\title{
Optimum response of functionally graded piezoelectric plates in thermal environments
}

\author{
HoSSEIN NOURMOHAMMADI, BASHIR BEHJAT* \\ Mechanical Engineering Faculty, Sahand University of Technology, P.O. Box 51335-1996, Tabriz, Iran
}

\begin{abstract}
In this article, the static response of the functionally graded piezoelectric (FGP) plates with piezoelectric layers (sandwich FGPM) is studied based on the first order shear deformation plate theory. The plate is under mechanical, electrical and thermal loadings and finite element method is employed to obtain the solution of the equation. All mechanical, thermal and piezoelectric properties, except Poisson ratio, obey the power law distribution through the thickness. By solving the governing equation, optimum value of power law index is investigated in each type of loading. The effects of different volume fraction index, layer arrangements, various boundary conditions and different loading types, are studied on the deflection of FGPM plate. It is inferred that, the correlations between the deflection, power law index and layer arrangement are completely different in the mechanical and thermal loading and the optimum value of the power law index should be selected in each case separately. This optimum values can be used as a design criterion to build a reliable sensors and actuators in thermal environments.
\end{abstract}

Keywords: finite element method; thermal loading; optimum power law index; sandwich FGPM

\section{Introduction}

In recent decades, the multilayered piezoelectric structures have been developed for use in different fields of engineering applications, such as sensors and actuators, health monitoring and vibration control [1-3]. Because of material properties of these structures at the layers interfaces, some problems such as residual stresses and delamination may occur in high temperature environments [4]. Also for multilayered piezoelectric structures with homogeneous material properties in each layer of the plate, concentration of stress, creep at high thermal environments and failure usually occur between the layers. To reduce such disadvantages, a new kind of materials was developed in which, one or several layers were made from functionally graded materials (FGMs) with different thermo-electro-mechanical properties changing softly through the thickness of the structure [5]. This type of materials is called FGPMs. There are many papers that deal with the behavior of such structures in thermal environments and some of them are mentioned here.

*E-mail: Behjat@sut.ac.ir
An exact 3-D analysis of FGPM plate for two types of boundary conditions (simply supported and four sides clamped) was performed by Zhong et al. [6]. They modeled a FGPM plate by considering that the mechanical and electric properties of the material obey power law distribution in the thickness direction. Lee [7] developed a linear layer-wise finite element formulation to study the static behavior of functionally graded piezoelectric bimorph actuators. This formulation was developed to study the displacement and stress response in thermo-electrical environments. The electrostatic behavior of FGPM cantilever beam based on the theory of elasticity has been studied by Xiang et al. [8]. They suggested a general formulation that can be used to compute the maximum deflection of a functionally graded piezoelectric actuator. Zhong et al. [9] presented an exact solution of a functionally graded piezo-thermo-electric simply supported rectangular plate. To obtain the solution, they used the double Fourier series. Yang et al. [10] investigated the static bending and dynamic response of actuators with various structures made of FGPMs under electro-thermal and mechanical loading considering Timoshenko beam theory. It is assumed that mechanical, electrical and thermal properties 
of the actuator, except Poisson ratio, are varying through the thickness direction due to power law distribution. Xiang et al. [11] performed the static analysis of the FGPM plate by using Airy stress function method and presented its response under electro-thermal loading. Alibeigloo [12] presented an analytical formula for thermo-electroelastic deformations of FGM cylindrical shells embedded within piezoelectric layers assuming axisymmetric behavior. Komeili et al. [13] studied the static response of FGPM beams in thermo-electromechanical environments. They compared the accuracy and the reliability of Euler Bernoulli, firstorder shear deformation and third-order shear deformation theory with each other. Wu et al. [4] presented three dimensional analysis of FGPM circular hollow sandwich cylinders in simply supported boundary conditions in thermal environments. Behjat et al. [14] used finite element method to investigate nonlinear behavior of functionally graded piezoelectric plates in electro-mechanical loading. Nechibvute et al. [15] presented a comprehensive study of the PZT ceramics and novel onelayer crystals in the selection of sensor or actuator substance. Li et al. [16] developed a functionally graded piezoelectric nanobeam model based on the Timoshenko beam theory and considering small scale effects by using a variational method. They solved bending and free vibration of the beam with simply supported boundary conditions. Nourmohammadi et al. [17] presented static bending response of the functionally graded piezoelectric material under mechanical, electrical, and thermal loads. They found that correlations between the deflection and the power law index are completely different under the mechanical and thermal loadings, which can be used to design structures in actuator or sensor state.

This article is devoted to the thermo-electromechanical analysis of FGPM plates with piezoelectric layers using finite element method. The bending response of the plate is presented based on the first order shear deformation plate theory (FSDT) under electrical, mechanical and thermal loadings. The pyroelectric propertes of the piezoelectric plate are considered. To obtain the response of the structure under various boundary conditions, the finite element method is used. By using mechanical and electrical equilibrium equations, governing variational forms of the equations for the FGPM plate are derived. The effects of different volume fraction index, layer arrangement and various loading conditions are studied on the deflection of FGPM plate with piezoelectric layers and the results are shown in a clear graphical form. This study investigates the effect of power law index on the behavior of multi-layer FGPM structures in thermal environments to find a new design criterion that can be used in the manufacturing of sensors and actuators.

\section{Geometry of the plate and prob- lem description}

\subsection{Functionally graded piezoelectric plates}

Various analytical and computational models for the spatial distribution of physical and mechanical properties of FGPMs have been proposed. However, the power law function is a simple type of such models [18-20]. It uses volume fraction of a material component to explain the spatial distribution of material properties through the thickness. For a functional material made of two materials, each property of the material can be expressed by the following equation:

$$
\begin{gathered}
V_{c}+V_{m}=1 \\
V_{c}=\left(\frac{z}{h}+\frac{1}{2}\right)^{n}, n \geqslant 0
\end{gathered}
$$

where $\mathrm{V}_{\mathrm{c}}, \mathrm{V}_{\mathrm{m}}$ are the volume fractions of bottom and top surface materials, respectively, and $n$ is the power law index. The effective material property is expressed by the following equation:

$$
\begin{aligned}
C_{e f f}= & C_{b o t}+\left(C_{t o p}-C_{b o t}\right)\left(\frac{z}{h}+\frac{1}{2}\right)^{n}= \\
& C_{b o t}+\left(C_{t o p}-C_{b o t}\right) V_{c}
\end{aligned}
$$

where $\mathrm{C}_{\text {eff }}$ is the effective material property, $\mathrm{C}_{\text {top }}$ is the top surface property of the plate, 
$\mathrm{C}_{\text {bot }}$ is the bottom surface property of the FGPM plate, and $V_{c}$ is the volume fraction of the material. In this paper, all mechanical, thermal and piezoelectric properties, except Poisson ratio, obey the power law distribution.

In this paper, a sandwich FGPM plate with dimensions of $50.8 \mathrm{~mm}^{3} \times 25.4 \mathrm{~mm}^{3} \times 5 \mathrm{~mm}^{3}$ with two piezoelectric layers $\left(\mathrm{h}_{1}=\mathrm{h}_{2}=0.1 \mathrm{~mm}\right)$ has been studied (Fig. 1). In this analysis, the effect of three types of layer arrangement on the static response of the plate under electro-thermomechanical loading has been studied. The FGPM plate consists of functionally graded materials. The upper surface of FGPM is PVDF-rich and lower surface is PZT-rich. Two types of boundary conditions are investigated in this paper. For convenience, the clamped boundary condition in the plate is termed by $\mathrm{C}$ and simply supported one is marked by S. Also three types of layer arrangement are considered in this article and the results for each type are compared with the other types of the layer arrangement (Fig. 1).
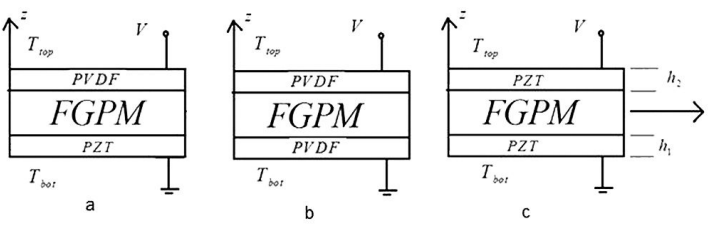

Fig. 1. Geometry of sandwich FGPM plate with three types of layer arrangement.

\section{Theoretical formulations}

\subsection{Constitutive relations}

Considering the linear thermo-piezoelectricity theory, the constitutive equations of a piezoelectric material can be expressed as [10]:

$$
\begin{gathered}
\sigma_{i j}=C_{i j k l} \varepsilon_{k l}-e_{i j k} E_{k}-\lambda_{i j} \theta \\
D_{l}=e_{i j} \varepsilon_{j}+k_{l k} E_{k}+p_{l} \theta
\end{gathered}
$$

Here $\sigma_{\mathrm{ij}}$ and $\epsilon_{\mathrm{kl}}$ are the stress and strain tensors, respectively, $\mathrm{D}_{1}, \mathrm{E}_{\mathrm{k}}$ are the electrical displacement and the electrical field vectors, $\mathrm{C}_{\mathrm{ijk}}$ is the elasticity matrix, $e_{i j}$ is the piezoelectric constant matrix, $\theta$ is the temperature gradient with respect to the reference value, $\lambda_{\mathrm{ij}}$ is the thermal expansion tensor, $\mathrm{p}_{1}$ is the pyroelectric vector and $\mathrm{k}_{\mathrm{lk}}$ is the dielectric permittivity coefficient matrix. $\lambda_{\mathrm{ij}}$ can be expressed by the following equation:

$$
\lambda_{i j}=C_{i j k l} \alpha_{k l}
$$

where $\mathrm{C}_{\mathrm{ijkl}}$ is the elasticity matrix and $\alpha_{\mathrm{kl}}$ is the thermal expansion coefficient vector.

\subsection{Displacements and strains}

According to the first order shear deformation theory of the plates, the displacement components in the plate can be expressed as [21]:

$$
\begin{aligned}
& u(x, y, z)=u_{0}(x, y)+z \beta_{x}(x, y) \\
& v(x, y, z)=v_{0}(x, y)+z \beta_{y}(x, y) \\
& w(x, y, z)=w_{0}(x, y)
\end{aligned}
$$

where the variables $\mathrm{u}, \mathrm{v}$ and $\mathrm{w}$ are displacements along the $\mathrm{x}, \mathrm{y}, \mathrm{z}$ axes, respectively. Also $\mathrm{u}_{0}, \mathrm{v}_{0}, \mathrm{w}_{0}$, denote the displacements of the mid-plane and $\beta_{\mathrm{x}}$, $\beta_{\mathrm{y}}$ are the rotations of a transverse normal about the $\mathrm{x}$ - and $\mathrm{y}$-axes.

The strain based on the displacements field in equation 3 is given by:

$$
\begin{aligned}
& \varepsilon_{x}=\frac{\partial u_{0}}{\partial x}+z \frac{\partial \beta_{x}}{\partial x}, \varepsilon_{y}=\frac{\partial v_{0}}{\partial y}+z \frac{\partial \beta_{y}}{\partial y} \\
& \gamma_{y z}=\frac{\partial w_{0}}{\partial y}+\beta_{y}, \gamma_{x z}=\frac{\partial w_{0}}{\partial x}+\beta_{x} \\
& \gamma_{x y}=\frac{\partial u_{0}}{\partial y}+\frac{\partial v_{0}}{\partial x}+z\left(\frac{\partial \beta_{x}}{\partial y}+\frac{\partial \beta_{y}}{\partial x}\right)
\end{aligned}
$$

By using Hook law, the constitutive equation of the FGPM material can be defined as:

$$
\left\{\begin{array}{l}
\sigma_{x} \\
\sigma_{y} \\
\tau_{x} z \\
\tau_{y} z \\
\tau_{x y}
\end{array}\right\}=[\bar{Q}]\left\{\begin{array}{c}
\varepsilon_{x} \\
\varepsilon_{y} \\
\varepsilon_{x} z \\
\varepsilon_{y} z \\
\varepsilon_{x} y
\end{array}\right\}
$$

where $\bar{Q}$ is: 


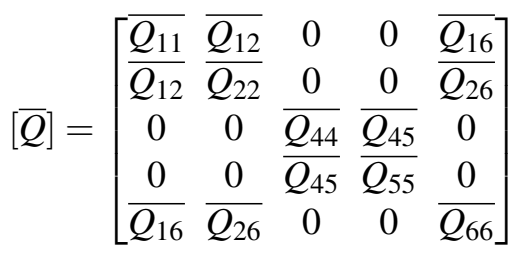

The governing equations of piezoelectric material are expressed in matrix form as following equation:

$$
\begin{aligned}
&\left\{\begin{array}{c}
\sigma_{x} \\
\sigma_{y} \\
\tau_{x} y
\end{array}\right\}= {\left[\begin{array}{lll}
\overline{Q_{11}} & \overline{Q_{12}} & \overline{Q_{16}} \\
\overline{Q_{16}} & \overline{Q_{22}} & \overline{Q_{26}} \\
Q_{66}
\end{array}\right]\left\{\begin{array}{c}
\varepsilon_{x} \\
\varepsilon_{y} \\
\gamma_{x y}
\end{array}\right\}-} \\
&\left\{\begin{array}{l}
\bar{e}_{31} \\
\bar{e}_{31} \\
\bar{e}_{31}
\end{array}\right\} E_{z}-\left[\begin{array}{l}
\bar{\lambda}_{x} \\
\bar{\lambda}_{y} \\
\bar{\lambda}_{x y}
\end{array}\right] \theta \\
&\left\{\begin{array}{c}
\tau_{x y} \\
\tau_{y z}
\end{array}\right\}=k_{5}\left[\begin{array}{ll}
\bar{Q}_{44} & \bar{Q}_{45} \\
\bar{Q}_{45} & \bar{Q}_{55}
\end{array}\right]\left\{\begin{array}{l}
\gamma_{x z} \\
\gamma_{y z}
\end{array}\right\}-\left[\begin{array}{ll}
\bar{e}_{15} & \bar{e}_{25} \\
\bar{e}_{14} & \bar{e}_{24}
\end{array}\right]\left\{\begin{array}{l}
E_{x} \\
E_{y}
\end{array}\right\}
\end{aligned}
$$

and:

$$
\begin{gathered}
D_{z}=\left[\begin{array}{lll}
\bar{e}_{31} & \bar{e}_{32} & \bar{e}_{36}
\end{array}\right]\left\{\begin{array}{l}
\varepsilon_{x} \\
\varepsilon_{y} \\
\gamma_{x y}
\end{array}\right\}+\bar{\varepsilon}_{33} \cdot E_{z}-\left[p_{z}\right] \theta \\
\left\{\begin{array}{l}
D_{x} \\
D_{y}
\end{array}\right\}=\left[\begin{array}{ll}
\bar{\varepsilon}_{11} & \bar{\varepsilon}_{12} \\
\bar{\varepsilon}_{12} & \bar{\varepsilon}_{22}
\end{array}\right]\left\{\begin{array}{l}
E_{x} \\
E_{y}
\end{array}\right\}+ \\
\\
{\left[\begin{array}{ll}
\bar{e}_{15} & \bar{e}_{25} \\
\bar{e}_{14} & \bar{e}_{24}
\end{array}\right]\left\{\begin{array}{l}
\gamma_{x z} \\
\gamma_{y z}
\end{array}\right\}-\left[\begin{array}{l}
p_{x} \\
p_{y}
\end{array}\right]\{\theta\}}
\end{gathered}
$$

It is worth to note that, in above equations the inplane and out-of-plane parameters are separated.

\subsection{Generalized equation in the varia- tional form}

The equilibrium equation of the plate is derived based on the conservation of momentum and electric charge equations. The variational form of the equilibrium equation of the piezoelectric plate can be defined as following equations [22]:

$$
\begin{aligned}
\delta u^{T} \psi_{u}= & -\int_{v}[\delta \varepsilon]^{T}[\sigma] d v+\int_{v} \delta \vec{u}^{T} \vec{b} d v+ \\
& \int_{\Gamma_{q}} \delta \vec{u}^{T} \vec{\tau} d \Gamma=0 \\
\delta \phi^{T} \psi_{e}=- & \int_{v} \delta E^{T} D d v+\int_{\Gamma_{q}} \delta \phi^{T} q d \Gamma=0
\end{aligned}
$$

The vectors $\psi_{\mathrm{u}}, \psi_{\mathrm{e}}$ are the difference between external and internal forces and charges, respectively. When mechanical and electric equilibrium occur, these vectors can be set to zero. $\vec{\tau}$ is the surface traction on the $\Gamma_{\mathrm{q}}$ and $\mathrm{q}$ is the electrical charge applied on the terminal bounding surface $\Gamma_{\mathrm{q}}$. Also $\mathrm{v}$ is the plate volume.

By substituting equation 8 in equation 13 and integrating in the thickness direction of the FGPM plate, and replacing the strain and electric field terms, the variational forms of the conservation equations are derived as:

$\int_{A_{0}}\left(\delta \overrightarrow{\varepsilon_{a}} T\left[C_{s}\right] \overrightarrow{\varepsilon_{a}}+\delta{\overrightarrow{\varepsilon_{b}}}^{T}\left[E_{e \theta}\right] \varepsilon_{e \theta}\right) d A+$

$\int_{A_{0}}\left(\delta u^{T} b^{T}+\delta \beta^{T} b^{T}\right) d A+\int_{\Gamma_{\text {tau }}} \delta \vec{u}^{T} \vec{\tau} d \tau=0$

$\int_{A_{0}}\left(\delta E\left[E_{G}\right] \overrightarrow{\varepsilon_{b}}+\delta E\left[G_{E}\right] \varepsilon_{e \theta}\right) d A+\int_{\Gamma_{q}} \delta \vec{\phi} \vec{q} d \Gamma=0$

where parameters $\overrightarrow{\epsilon_{\mathrm{a}}}, \overrightarrow{\epsilon_{\mathrm{b}}}, \epsilon_{\mathrm{e} \theta}, \mathrm{C}_{\mathrm{s}}, \mathrm{E}_{\mathrm{e} \theta}, \mathrm{E}_{\mathrm{G}}, \mathrm{G}_{\mathrm{E}}$ are defined as:

$$
\begin{aligned}
& \overrightarrow{\varepsilon_{a}}=\left[\begin{array}{l}
\varepsilon^{0} \\
k^{0} \\
\varepsilon_{s}^{0}
\end{array}\right] \varepsilon_{b}=\left[\begin{array}{l}
\delta \varepsilon^{0} \\
\delta k^{0}
\end{array}\right] \overrightarrow{\varepsilon_{e \theta}}=\left[\begin{array}{c}
E \\
\theta
\end{array}\right]
\end{aligned}
$$

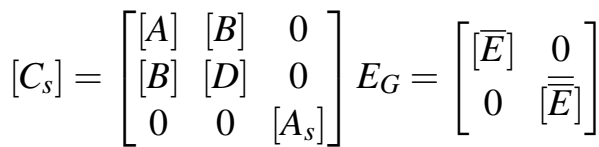

$$
\begin{aligned}
& G_{E}=\left[\begin{array}{cc}
{[G]} & 0 \\
0 & {[T]}
\end{array}\right] E_{e \theta}=\left[\begin{array}{cc}
{[\bar{E}]} & -[\bar{\Theta}] \\
{[\overline{\bar{E}}]} & -[\overline{\bar{\Theta}}]
\end{array}\right]
\end{aligned}
$$

In the above equations, A, B, D are extensional, bending-extensional coupling and bending stiffness matrices, respectively. $\mathrm{A}_{\mathrm{s}}$ is the shear stiffness matrix; $\overline{\mathrm{E}}, \overline{\overline{\mathrm{E}}}$ are in-plane and out-of-plane piezoelectric matrices of each layer of the plate; $G$ is the electric permittivity matrix of each layer of the plate; $\bar{\Theta}, \overline{\bar{\Theta}}$ are the in-plane and out-of-plane thermal expansion matrices and $\mathrm{T}$ is the FGPM pyroelectric matrix. These parameters are defined by the following expressions:

$$
\begin{gathered}
{[\bar{E}]=[\bar{e}] \cdot\left(z_{k+1}-z_{k}\right),[\overline{\bar{E}}]=\frac{1}{2}[\bar{e}] \cdot\left(z_{k+1}^{2}-z_{k}^{2}\right)} \\
{[G]=[\bar{\varepsilon}] \cdot\left(z_{k+1}-z_{k}\right),[\bar{\Theta}]=[\bar{\lambda}] \cdot\left(z_{k+1}-z_{k}\right)} \\
{[\overline{\bar{\Theta}}]=\frac{1}{2}[\bar{\lambda}] \cdot\left(z_{k+1}^{2}-z_{k}^{2}\right),[T]=[\bar{p}] \cdot\left(z_{k+1}-z_{k}\right)(17)}
\end{gathered}
$$




\subsection{Finite element model}

The finite element method is employed to solve the equation of the FGPM plate. The plate has been modeled with an 8-node rectangular element that has 7 degree of freedom for each node, the displacements, electric potential and temperature. The field variables can be expressed in terms of nodal variables, as follows:

$$
\begin{aligned}
& u_{0}(x, y)=\sum_{i=1}^{8} N_{i}(x, y) u_{0 i} \\
& v_{0}(x, y)=\sum_{i=1}^{8} N_{i}(x, y) v_{0 i} \\
& w_{0}(x, y)=\sum_{i=1}^{8} N_{i}(x, y) w_{0 i} \\
& \beta_{x}(x, y)=\sum_{i=1}^{8} N_{i}(x, y) \beta_{x i} \\
& \beta_{y}(x, y)=\sum_{i=1}^{8} N_{i}(x, y) \beta_{y i} \\
& \phi(x, y)=\sum_{i=1}^{8} N_{i}(x, y) \phi_{i} \\
& \theta(x, y)=\sum_{i=1}^{8} N_{i}(x, y) \theta
\end{aligned}
$$

where $\mathrm{N}_{\mathrm{i}}$ is the interpolation function and $\mathrm{u}, \mathrm{v}, \mathrm{w}$, $\beta_{\mathrm{x}}, \beta_{\mathrm{y}}, \varphi, \theta$ are the nodal values of displacements, rotations, electric potential and temperature in the element. By using above mentioned equations and displacement-strain relation (equation 8), the final form of the strain-displacement relation can be obtained as follows:

$$
[\vec{\varepsilon}]=\left[B_{u}\right] \vec{u}=\left[R_{a}\right] \vec{u}+z\left[R_{b}\right] \vec{u}+\left[R_{s}\right] \vec{u}
$$

where $\left[\mathrm{R}_{\mathrm{a}}\right],\left[\mathrm{R}_{\mathrm{b}}\right],\left[\mathrm{R}_{\mathrm{s}}\right]$ are strain operators based on the strain-displacement relations.

Combining equation 17 with equation 13 and integrating through the area, the final equation of equilibrium in the matrix form can be obtained as:

$$
\begin{gathered}
{\left[K_{d d}\right][\vec{U}]+\left[K_{d e}\right]\left[\overrightarrow{\varphi^{s}}\right]+\left[K_{d e}\right]\left[\overrightarrow{\varphi^{a}}\right]-\left[K_{d \theta}\right][\vec{\theta}]=[F]} \\
{\left[K_{e d}\right][\vec{U}]-\left[K_{e e}\right]\left[\overrightarrow{\varphi^{s}}\right]-\left[K_{e e}\right]\left[\varphi^{a}\right]+\left[K_{e \theta}\right][\vec{\theta}]=0}
\end{gathered}
$$

where submatrices $\mathrm{K}_{\mathrm{dd}}, \mathrm{K}_{\mathrm{de}}, \mathrm{K}_{\mathrm{ee}}$ denote the elastic, piezoelectric and permittivity stiffness matrices; $K_{d \theta}$ and $K_{e \theta}$ are the coupled thermal expansion and pyroelectric stiffness matrices of the plate. Also, $U$ is the nodal parameters vector that consists of five generalized displacement degrees of freedom per node; the three displacements $(\mathrm{u}, \mathrm{v}, \mathrm{w})$ and two rotations $\left(\beta_{\mathrm{x}}, \beta_{\mathrm{y}}\right)$. Also $\varphi$ is the nodal electric potential vector and $\theta$ is the applied nodal temperature vector. Superscripts s and a indicate the partitioned submatrices in accordance with the sensory (free) and active (actuator) electric potential components, respectively.

Sensory electric potential using equation 18 is expressed as follows:

$\left[\overrightarrow{\varphi^{s}}\right]=\left[K_{e e}\right]^{-1}\left(\left[K_{e d}\right][\vec{U}]+\left[K_{e \theta}\right][\vec{\theta}]-\left[K_{e e}\right]\left[\overrightarrow{\varphi^{a}}\right]\right)$

Combining equation 22 and equation 21, the following equation in matrix form can be obtained:

$$
\begin{aligned}
& \left(\left[K_{d d}\right]+\left[K_{d e}\right]\left[K_{e e}\right]^{-1}\left[K_{e d}\right]\right)[\vec{U}]= \\
& {[F]+\left[K_{d \theta}\right][\vec{\theta}]-\left[K_{d e}\right]\left[K_{e e}\right]^{-1}\left[K_{e \theta}\right][\vec{\theta}]+} \\
& {\left[K_{d e}\right]\left[K_{e e}\right]^{-1}\left[K_{e e}\right]\left[\overrightarrow{\varphi^{a}}\right]-\left[K_{d e}\right]\left[\overrightarrow{\varphi^{a}}\right]}
\end{aligned}
$$

\section{Numerical results and discus- sion}

\subsection{Comparison study}

In this section, to verify the proposed model, two different examples are presented for the static analysis of composite piezoelectric plates and FGM plate with two piezoelectric layers. The first example is presented to validate the piezoelectric and thermal behavior of the model. The other example is provided to examine the functionally graded behavior of the plate.

Example 1. In this example a plate with dimensions of $25.4 \mathrm{~mm}^{3} \times 25.4 \mathrm{~mm}^{3} \times 1.27 \mathrm{~mm}^{3}$ and with the layers of $[0 / 90 / \pm 45 / \mathrm{p}]_{\mathrm{s}}$ composed of carbon/graphite with attached piezoceramic layer under temperature gradient is investigated and the results compared with the data reported by Bansal et al.[23] (Fig. 2). The plate is clamped in one side and is subjected to $100{ }^{\circ} \mathrm{C}$ temperature gradient. 
Fig. 3 depicts the dimensionless centerline deflection of the plate. It is seen that, the results obtained in this paper have a good agreement with the results reported by Bansal et al. [23].

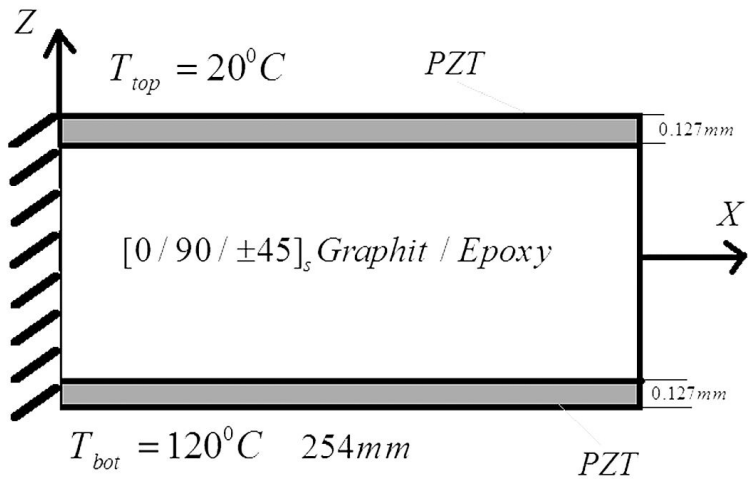

Fig. 2. Geometry of a clamped composite plate with attached piezoelectric layer [23].

Example 2. In the other case, an FGM plate with piezoelectric layers has been studied. The plate is clamped on one side and a uniform mechanical load of $\mathrm{q}=100 \mathrm{kN} / \mathrm{m}^{2}$ and actuator voltage $40 \mathrm{~V}$ is applied at the top surface of the plate. The middle layer of the plate is made of FGM and has a thickness of $5 \mathrm{~mm}$ and the upper and lower layers are made of piezoelectric material with the thickness of $0.1 \mathrm{~mm}$. The FGM layer is composed of zirconia and aluminum. Fig. 4 depicts the centerline deflection of the cantilever FGM plate. From this figure, it is inferred that the results obtained in this paper have a good agreement with the results reported by Dai et al. [24].

In this paper, thermal, electrical and mechanical analysis of the sandwich FGPM plate has been performed. The plate consists of one FGPM layer embedded with pure piezoelectric layers. The FGPM plate consists of PVDF-rich at upper surface and PZT-rich at lower surface. Material properties of the plate are listed in Table 1. In this article, "thermal environment" means a condition that the structure is subjected to temperature gradient throughout the thickness of the plate. The convergence study of sandwich FGPM response has been done for three boundary condition. Fig. 5 shows the convergence of deflection in the center point of the plate for three kinds of boundary conditions.

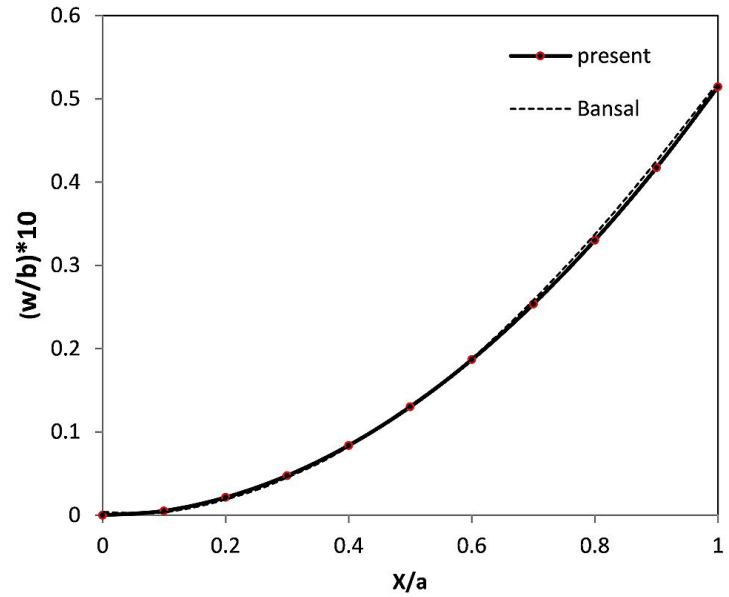

Fig. 3. Centerline defection of composite plate with piezoelectric layer under $100{ }^{\circ} \mathrm{C}$ thermal gradient.

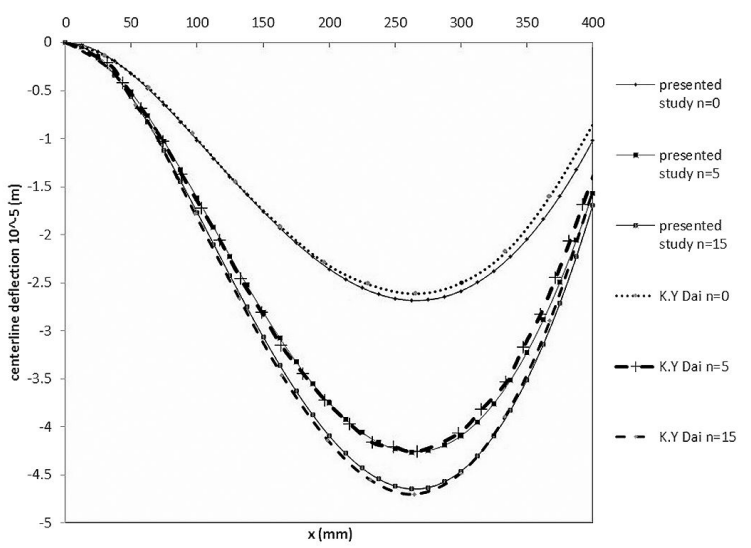

Fig. 4. Centerline deflection of the cantilever FGM plate subjected to mechanical and electrical load.

It is observed that, the results are converged by using 50 elements.

\subsection{FGPM plate with piezoelectric layers under mechanical and electrical loading}

In this section, a FGPM plate with three kinds of layers (Fig. 1) at the top and bottom surfaces that is subjected to uniform mechanical loading $\left(\mathrm{q}=10 \mathrm{kN} / \mathrm{m}^{2}\right)$ has been studied. Here, the effect of the power law index $n$ on the deflection of FGPM plate is studied. Fig. 6 shows the centerline deflection of FGPM plate with three kinds of layer 
Table 1. Material properties of PVDF and PZT [25].

\begin{tabular}{ccc}
\hline Properties & PVDF & PZT \\
\hline \hline $\mathrm{E}$ & $2 \mathrm{GPa}$ & $60 \mathrm{GPa}$ \\
$\mathrm{v}$ & 0.3333 & 0.3333 \\
$\mathrm{G}$ & $0.75 \mathrm{GPa}$ & $22.5 \mathrm{GPa}$ \\
$\alpha$ & $1.2 \times 10^{-4} \frac{1}{{ }^{\circ} \mathrm{C}}$ & $1.2 \times 10^{-6} \frac{1}{{ }^{\circ} \mathrm{C}}$ \\
$\mathrm{k}$ & $1 \times 10^{-10} \frac{\mathrm{F}}{\mathrm{m}}$ & $150 \times 10^{-10} \frac{\mathrm{F}}{\mathrm{m}}$ \\
$\mathrm{d}_{31}=\mathrm{d}_{32}$ & $2.5 \times 10^{-11} \frac{\mathrm{C}}{\mathrm{N}}$ & $-17.5 \times 10^{-11 \frac{\mathrm{C}}{\mathrm{N}}}$ \\
$\mathrm{p}_{3}$ & $2.5 \times 10^{-5} \frac{\mathrm{C}}{{ }^{\circ} \mathrm{Cm}^{2}}$ & $-75 \times 10^{-5} \frac{\mathrm{C}}{{ }^{\circ} \mathrm{Cm}^{2}}$ \\
\hline
\end{tabular}

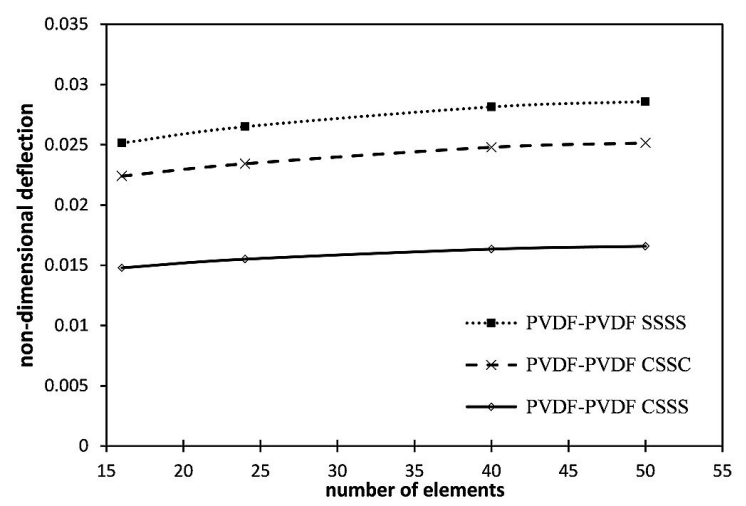

Fig. 5. Convergence study of sandwich FGPM plate versus the number of elements.

arrangement under mechanical loading with CSSS and CSSC boundary conditions and for power law index $\mathrm{n}=15$.

Fig. 7 and Fig. 8 compare the response of the plate with three kinds of layer arrangement under mechanical and electrical loadings. Fig. 7 depicts the maximum deflection for the three kinds of layer arrangement under mechanical loading. According to this figure, it is observed that by increasing of power law index the deflection of the plate decreases. This can be explained by the increase in the stiffness of FGPM plate. It is presumed that, the layer arrangement $b$ has maximum deflection due to lower stiffness than others and the layer arrangement $\mathrm{c}$ has minimum deflection due to higher stiffness than other cases.

f Fig. 8 shows maximum deflection for three kinds of layer arrangement under electromechanical loading. This figure shows that the layer arrangement a has good response to volt-

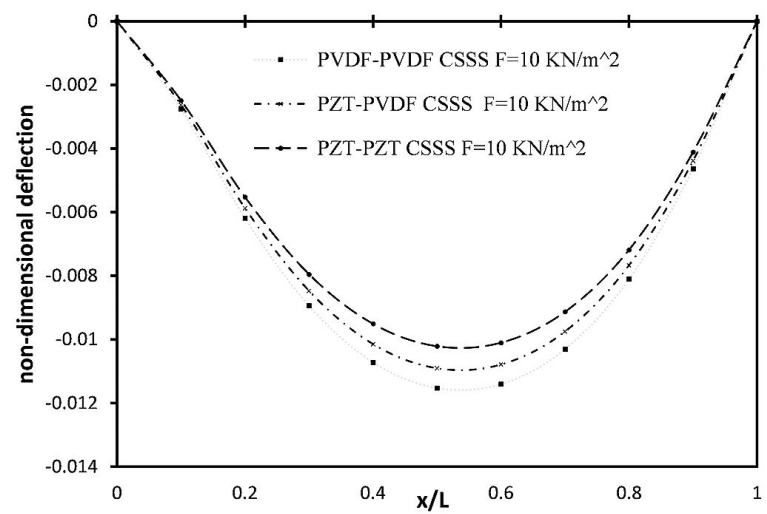

(a)

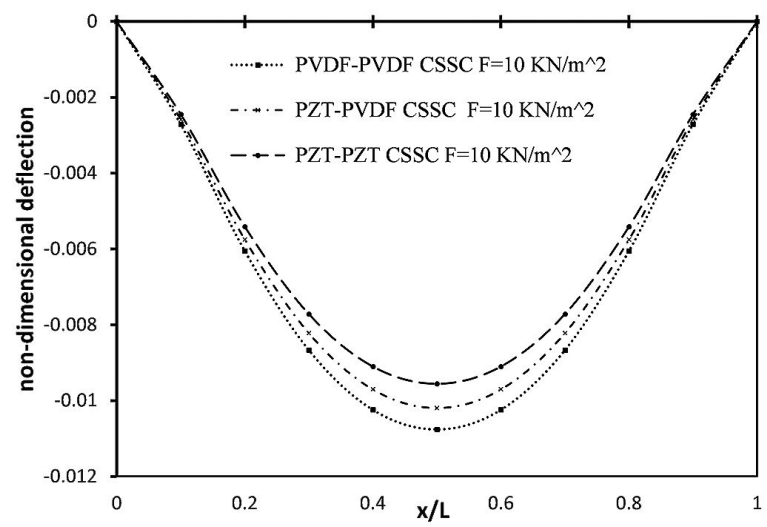

(b)

Fig. 6. The centerline deflection of FGPM plate with three kinds of layers under mechanical loading: (a) CSSS, and (b) CSSC.

age and reduces the deflection of the plate more than the other cases. This phenomenon is related to the effect of voltage on deflection, considering that the sign of piezoelectric constants of upper layer (PVDF) and lower layer (PZT) are different. It means that when a voltage is applied to the plate, the top layer becomes shorter and the bottom layer becomes longer and it helps to decrease the deflection caused by mechanical loading.

\subsection{FGPM plate with piezoelectric layers under thermal loading}

In this section, the plate is exposed to thermal loading. The plate is under $10{ }^{\circ} \mathrm{C}$ to $100{ }^{\circ} \mathrm{C}$ thermal loading that means the upper and lower layer 


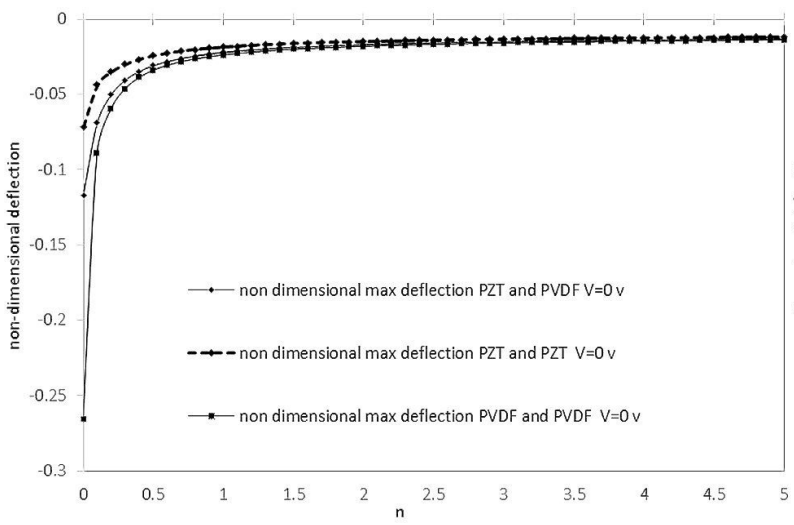

(a)

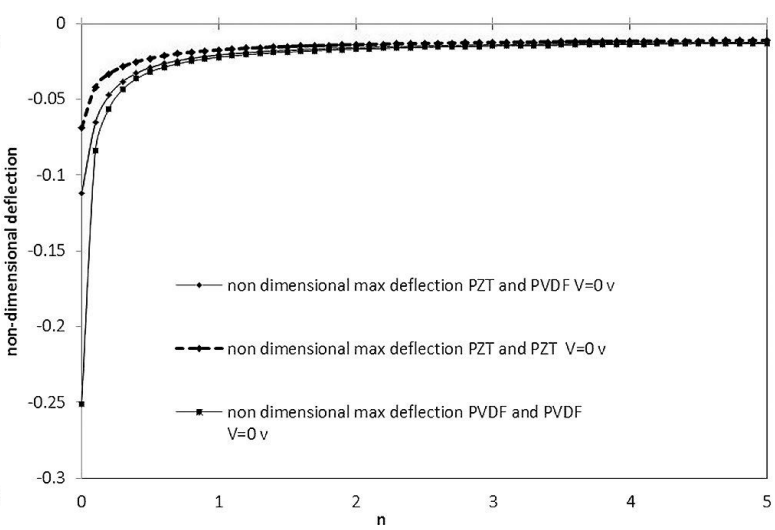

(b)

Fig. 7. The maximum deflection of FGPM plate with three kinds of layers under mechanical loading for various power law indexes, (a) CSSS, and (b) CSSC.

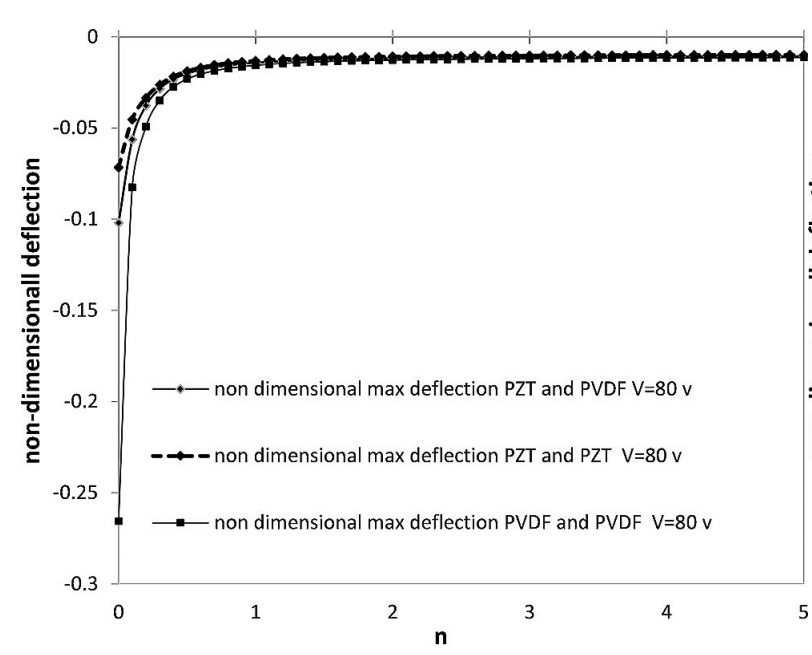

(a)

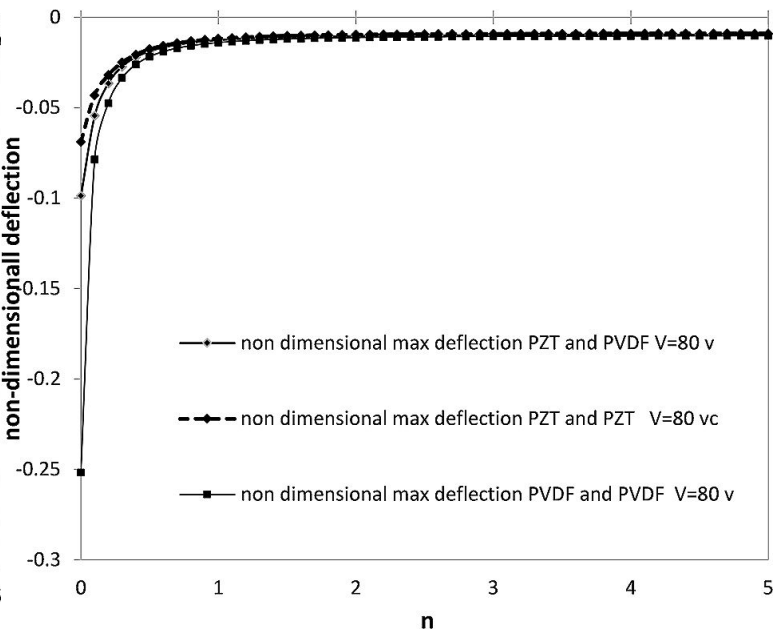

(b)

Fig. 8. The maximum deflection of the FGPM plate with three kinds of layers under electro-mechanical loading for various power law indexes: (a) CSSS, and (b) CSSC.

temperature is $\mathrm{T}_{\text {top }}=10{ }^{\circ} \mathrm{C}$ to $100{ }^{\circ} \mathrm{C}$ and $\mathrm{T}_{\text {bot }}=0{ }^{\circ} \mathrm{C}$, respectively.

Fig. 9 shows the centerline deflection of the FGPM plate with three kinds of layer arrangement, for CSSS and CSSC boundary conditions, that is exposed to $30{ }^{\circ} \mathrm{C}$ thermal gradient.

It is worth to note that a difference in the thermal expansion coefficients of two types of materials causes deflection under uniform thermal loading. When a sandwich FGPM plate is under uniform thermal loading, due to $\alpha_{1}$, the plate experiences in-plane displacements.
If this displacement is changing through the thickness, the lateral deflection occurs. Also, when a sandwich FGPM plate is under gradient thermal loading due to difference of temperature through the thickness, the plate will experience different in-plane displacements through the thickness, so deflection will occur. Because of the combination of these two mechanisms in the thermal loadings, the behavior of sandwich FGPM will be different.

Fig. 10 shows the maximum deflection of the FGPM plate with three kinds of layer 


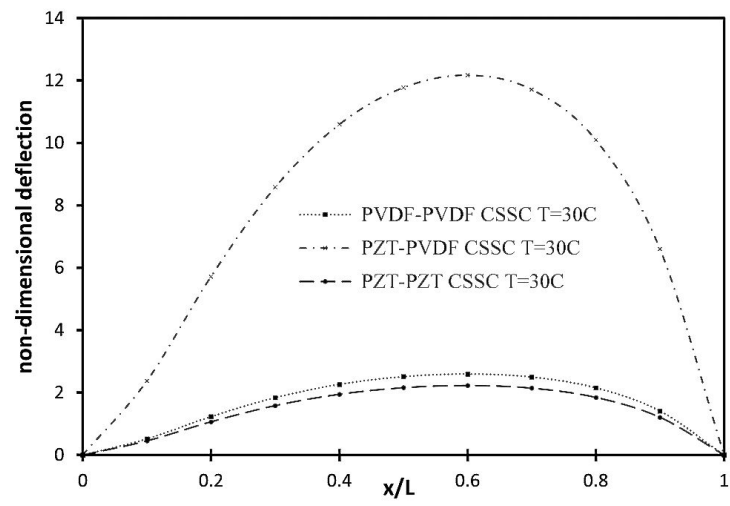

(a)

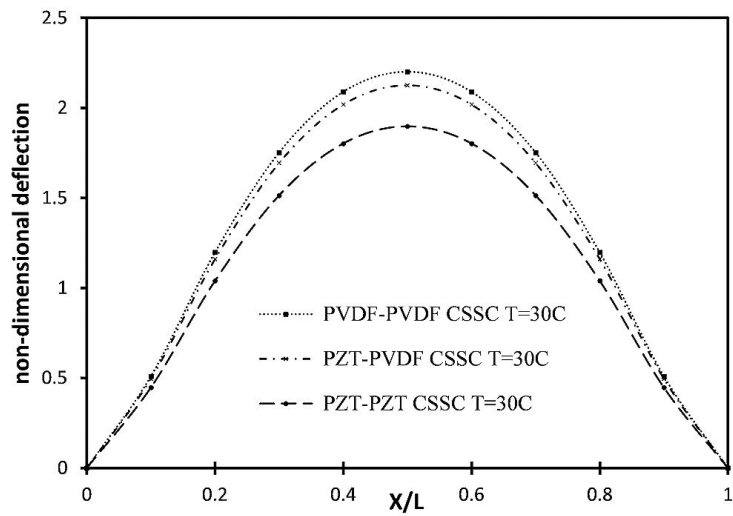

(b)

Fig. 9. The centerline deflection of FGPM plate with three kinds of layers under thermal loading: (a) CSSS, (b) CSSC.

arrangement for CSSS and CSSC boundary conditions, that is exposed to thermal loading. From this figure it is inferred that, with increasing the power index $n$ the deflection of the plate increases and then decreases. The reason of this behavior can be explained by the difference in the coefficients of thermal expansion and Young modulus of PZT and PVDF. As previously mentioned, the difference between thermal expansions causes the plate to experience different in-plane displacements through the thickness under thermal loading and this causes the deflection of the FGPM plate in lateral direction. It can be seen that, in the layer arrangement a the maximum plate deflection occurs for $\mathrm{n}=0.4$ at the both boundary conditions. This behavior also happens in layer arrangements $\mathrm{b}$ and $\mathrm{c}$ and maximum plate deflection occurs for $\mathrm{n}=0.5$ and $\mathrm{n}=0.9$, respectively. These values can be an optimum point if we use the structure as an actuator.

Fig. 11 compares the response of the plate to three kinds of layer arrangements under thermal loading. This figure shows the maximum deflection of three kinds of layer arrangement under thermal loading. It is inferred from this figure that the layer arrangement a has maximum deflection due to high thermal expansion coefficient and the layer arrangement $\mathrm{c}$ has minimum deflection due to higher stiffness than others. By using this optimum values in the design of sensors, efficient performance of this materials can be achieved.

\subsection{FGPM plate with piezoelectric layers under electrical loading}

Fig. 12 shows maximum deflection of FGPM plate with three kinds of layer arrangement subjected to $80 \mathrm{~V}$ electrical loading. It is seen in the layer arrangement a that the maximum plate deflection occurs for $\mathrm{n}=0$ at the both boundary conditions and it is due to PZT and PVDF at the top and bottom of the plate. But in layer arrangements $\mathrm{b}$ and $\mathrm{c}$, maximum plate deflection occurs for $n=0.3$ and $n=0.8$, respectively. In case of $\mathrm{b}$ and $\mathrm{c}$ if $\mathrm{n}=0$, then the out-of plane piezoelectric matrix becomes zero, so the deflection of sandwich FGPM became zero. But in case of a, when $n=0$ because of PZT and PVDF layers at the top and bottom of FGMP, the out-of-plane piezoelectric matrix $\overline{\overline{\mathrm{E}}}$ is non zero, so the deflection of sandwich FGPM plate becomes non zero. From Fig. 12 it is inferred that, with increasing the power index $n$ the deflection of the plate (case $b$ and case c) increases and then decreases. The reason of this behavior can be explained by the difference in the piezoelectric coefficients and Young moduli of PZT and PVDF and interaction between piezoelectric constant and Young modulus of the sandwich plate. The difference between piezoelectric coefficients causes the plate to experience different in-plane displacements through the thickness in electrical loading and this causes the deflection of the sandwich FGPM plate in lateral direction. 
$P Z T-P V D F$
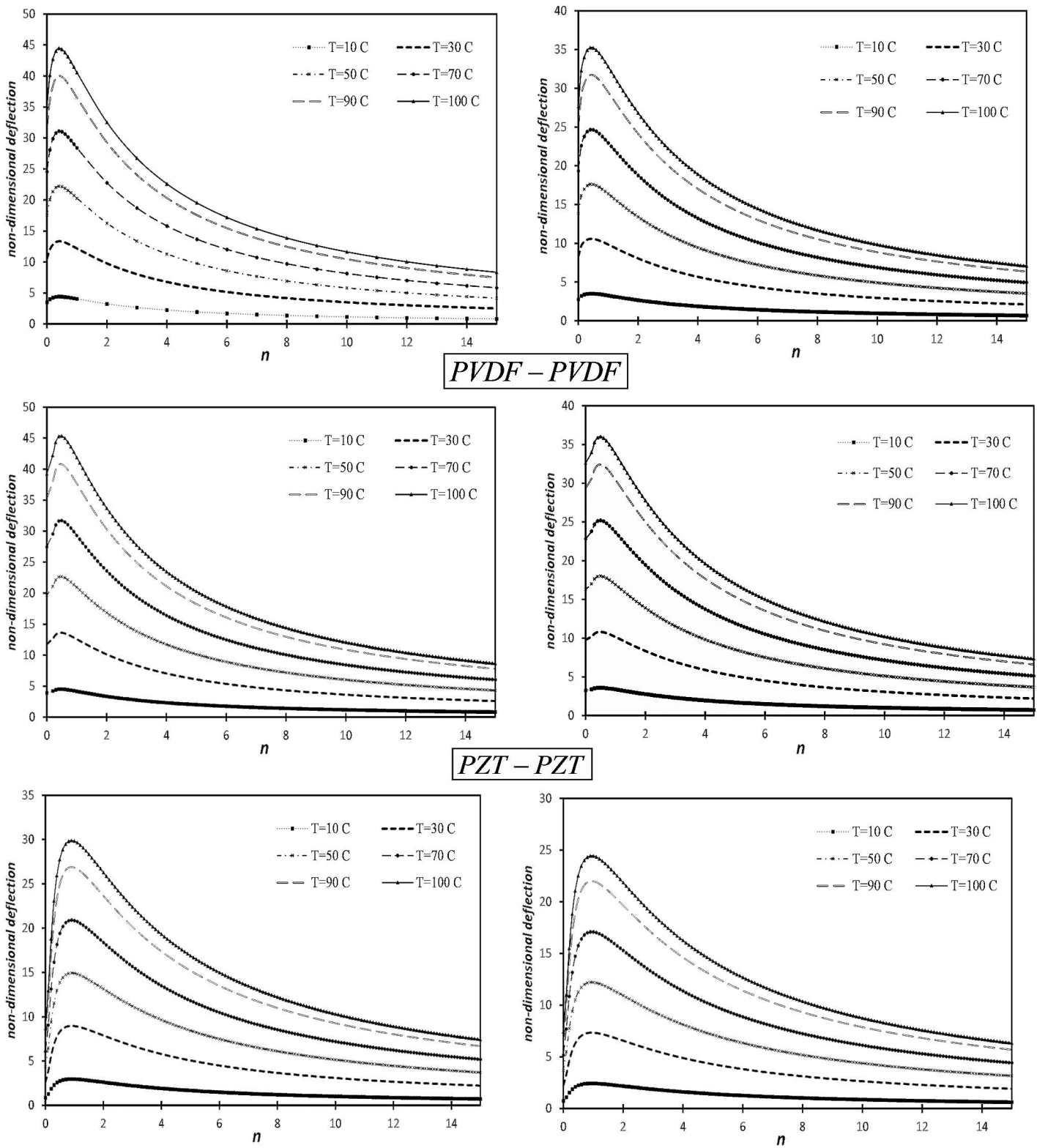

Fig. 10. Maximum deflection of FGPM plate with three kinds of layers under $10{ }^{\circ} \mathrm{C}$ to $100{ }^{\circ} \mathrm{C}$ temperature gradient: (a) CSSS and (b) CSSC.

With increasing $\mathrm{n}$, Young modulus and the piezoelectric constants of the sandwich FGPM plate increase. By increasing Young modulus, the deflection decreases, but by increasing the piezoelectric constant, the deflection increases, and these factors cause a maximum point for deflection in FGPM plate under electrical loading. These values can be selected as an optimum point if we use the structure as an actuator.

\section{Conclusions}

In this article, the bending response of multilayered FGPM plate is studied based on the first order shear deformation theory under mechanical, 


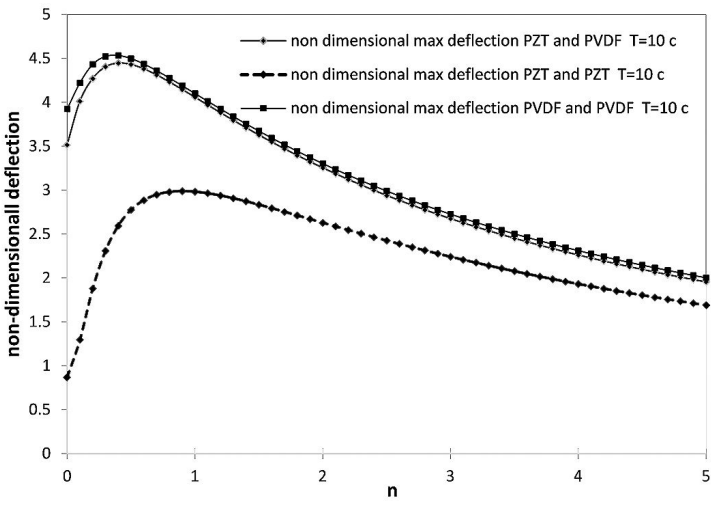

(a)

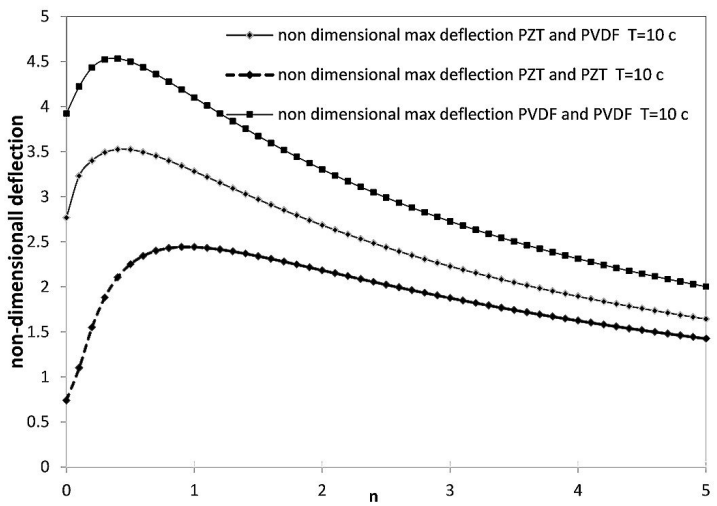

(b)

Fig. 11. Maximum deflection of FGPM plate with three kinds of layers under thermal loading for various power law indexes: (a) CSSS, (b) CSSC.

electrical and thermal loadings. By using mechanical and electrical equilibrium equations, the governing variational forms of the equations for the FGPM plate are derived and the finite element method is used to solve the problem. The effects of different volume fraction index, layer arrangement, various loading conditions and boundary conditions on the deflection of FGPM plate are studied. In three kinds of layer arrangement, the case a has good response to applied voltage; when a voltage is applied to the plate it shows larger decrease in deflection than other cases. Under the thermal loading at specific power law index, the deflection becomes maximum at each layer arrangement and it can be used to design sensors and actuators in thermal environments to reduce the effect of thermal changes

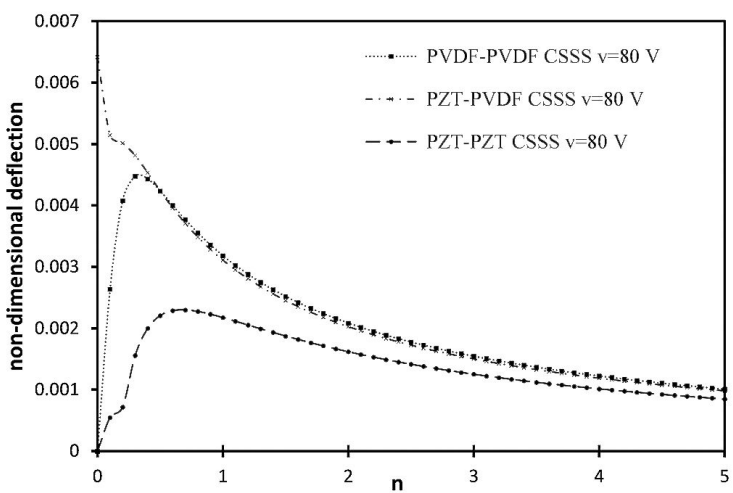

(a)

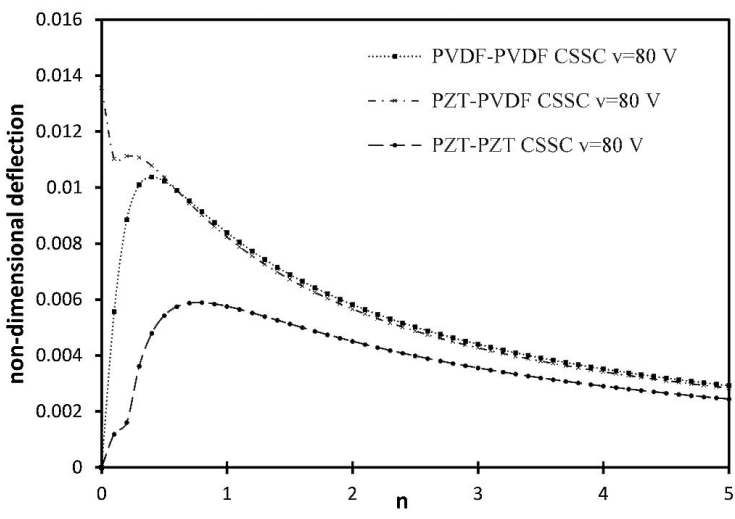

(b)

Fig. 12. Maximum deflection of FGPM plate with three kinds of layers under $80 \mathrm{~V}$ electrical loading: (a) CSSS, and (b) CSSC.

in sensors and actuators response. Under the thermal loading, layer arrangement case $b$ is more sensitive than other, due to greater thermal expansion coefficient and case c shows more stationary behavior than other cases. By using this feature, the efficient point of power law index in sensors and actuators can be found to design such structures.

\section{References}

[1] Branco P.J.C., Dente J.A. Smart Mater. Struct., 4 (2004), 631.

[2] Gu H., Moslehy Y., Sanders D., SonQ G., Mo Y.L., Smart Mater. Struct., 6 (2010), 65026.

[3] Km L., XQ H., Ng T.Y., Sivashanker S., Int. J. Numer. Meth. Eng., 11 (2001), 1253.

[4] Wu C.P., JiAng R.- Y., J. Intel. Mat. Syst. Str., 7 (2011), 691. 
[5] Wu X.H., Shen Y.P., Tian X.-G., Int. J. Solids Struct., 20 (2002), 5325.

[6] Zhong Z., Shang E.T., Int. J. Solids Struct., 20 (2003), 5335.

[7] LEE H.J., J. Intel. Mat. Syst. Str., 4 (2005), 365.

[8] Xiang H.J., Shi Z., J. Intel. Mat. Syst. Str., 7 (2007), 719.

[9] Zhong Z., Shang E.T., J. Intel. Mat. Syst. Str., 8 (2005), 643.

[10] Yang J., Xiang H.J., Smart Mater. Struct., 3 (2007), 784.

[11] Xiang H.J., Shi Z.F., Eur. J. Mech. A-Solid., 2 (2009), 338.

[12] Alibeigloo A., Compos. Struct., 2 (2011), 961.

[13] Komeili A., Akbarzadeh A.H., Eslami M.R., Adv. Mech. Eng., 3 (2011), 153731.

[14] Behjat B., Khoshravan M., Compos. Struct., 3 (2012), 874.

[15] Nechibvute A., Chawanda A., Luhanga P., ISRN Mater. Sci., 2012 (2012), 1.
[16] Li Y.S., Feng W.J., Cai Z.Y., Compos. Struct., 115 (2014), 41.

[17] Nourmohammadi H., Behjat B., J. Intel. Mat. Syst. Str., 16 (2016), 2249.

[18] Yildirim B., Dag S., Erdogan F., Int. J. Fracture, 4 (2005), 371.

[19] REdDY J., Int. J. Numer. Meth. Eng., 3 (2000), 663.

[20] NG T.Y., LAM K.Y., LIEW K.M., REDDY J.N., Int. J. Solids .Struct., 8 (2001), 1295.

[21] Reddy J.N., Eng. Struct., 7 (1999), 568.

[22] Varelis D., Saravanos D.A., Int. J. Numer. Meth. Eng., 1 (2008), 84.

[23] Bansal A., Ramaswamy A., J. Intel. Mat. Syst. Str., 5 (2002), 291.

[24] Dai K.Y., LiU G.R., Han X., Lim K.M., Comput. Struct., 18 (2005), 1487.

[25] Blandford G.E., Tauchert T.R., Du Y., Compos. Part B-Eng., 1 (1999), 51.

Received 2017-01-24

Accepted 2017-08-21 